\title{
Archimedes' Principle and Gravitation as a Spontaneous Process
}

\author{
Giancarlo Cavazzini ${ }^{1}$ \\ ${ }^{1}$ Consiglio Nazionale delle Ricerche, Istituto di Geoscienze e Georisorse, Via G. Gradenigo, Padova, Italy \\ Correspondence: Giancarlo Cavazzini, CNR Istituto di Geoscienze e Georisorse, Via G. Gradenigo, 6, 35131 - \\ Padova, Italy. Tel: 39-049-827-9137. E-mail: giancarlo.cavazzini@igg.cnr.it \\ Received: February 14, 2020 \\ Accepted: April 2, 2020 \\ Online Published: May 8, 2020 \\ doi:10.5539/apr.v12n3p1 \\ URL: http://dx.doi.org/10.5539/apr.v12n3p1
}

\begin{abstract}
A different physical interpretation of floating of bodies - the so-called "Archimedes' Principle" - relates the gravitational process to its essential characteristic: its spontaneity. Gravity is a spontaneous physical process similar, for example, to the process we interpret as 'heat transfer' between bodies at different temperature; similar to the process which occurs when masses of gas in contact with each other are at different pressures; similar to the process we call 'friction'. Gravitation has all the characteristics shown by other spontaneous processes, and, like them, its cause is disequilibrium, between matter in contact, in a 'quality' of that matter. In the case of gravitation, the quality of matter is related to its intensive property we call 'density'.

Just as the process we call 'heat transfer' is the spontaneous process by means of which the Universe reduces and eliminates disequilibria that exist between its parts, due to differences in 'temperature', what we call 'gravitation' is the spontaneous process by means of which the Universe reduces and eliminates disequilibria between its parts, due to differences in 'density'. This is achieved by moving bodies. Ceteris paribus, the process moves a body in the direction in which the difference in density between the matter of the body and the matter of the fluid surrounding the body is reduced.
\end{abstract}

Keywords: Archimedes' Principle, Gravitation, Spontaneous Processes

\section{[...] All things come about by strife. \\ Heraclitus of Ephesus}

That one body may act upon another at a distance through vacuum, without a mediation of anything else, by and through which their action and force may be conveyed from one to another is to me so great an absurdity, that I believe no man who was in philosophical matter a competent faculty of thinking, can ever fall into it.

\section{Introduction}

\section{Newton}

From the first, ancient interpretations to the most recent ones, gravitation is still extensively debated. Why is gravity so difficult to understand? Perhaps this physical process has been more interpreted than observed. In order to study a process properly, we should start from 'pure' observation, providing a description of the process while carefully avoiding any interpretation of it. In particular, we should avoid interpretation in giving it a 'definition'. Interpretation is necessary to Science, but it can be misleading.

However, despite the existence of this simple, well-known norm, common descriptions/definitions of gravitation are not real descriptions, because they contain a set of hypotheses. As an example, we give below the British Dictionary's definition of 'Gravitation':

Gravitation is the force of attraction that bodies exert on one another as a result of their mass. 
A first hypothesis is that the weight/movement/acceleration of a material body is caused by a 'physical entity' 'acting on the body' called 'force', thus considering the 'gravitational force' somewhat alien to the body itself.

A second hypothesis is that this 'force' is a 'force of attraction' between bodies, therefore 'working at a distance'. This interpretation is usually attributed to Isaac Newton, although the great scholar vehemently wrote against it (Newton, 1693).

A third hypothesis is that this 'force of attraction' originates because bodies 'have mass' (the 'mass' of a body was defined as the product between the volume and 'density' of the matter constituting the body).

Evidently, this is not a right way to describe a process. However, how can we describe gravitational process without introducing any interpretation? What can be said for sure about gravitational action? It can be said for sure that this physical process occurs spontaneously.

Neither the theory of the 'force of attraction at a distance' (Newton, 1687; Mach, 1912), nor the General Theory of Relativity (Einstein, 1916), nor the more recent theories - as, for example, the MOND theory (Milgrom 1983a; 1983b) and the MOG theory (Moffat and Rahvar, 2013) - considered the fact that this physical process occurs spontaneously. The essential characteristic of gravitation was unseen or neglected.

This paper discusses gravitation from the viewpoint of the spontaneous processes. First, the importance of the physical interpretation of the phenomenon of floating of bodies to understand gravitation is discussed (Cavazzini, 2018; 2019). Then, spontaneous processes are considered, in order to identify common characteristics. Lastly, it is shown that gravitation has all the characteristics exhibited by the other spontaneous processes.

\section{Archimedes' Principle and Hypotheses about Gravitational Mechanical Action}

Let us consider a material body, for example a piece of wood. Let this body free and completely immersed in a fluid body, for example immersed in air. We see that the body is not in mechanical equilibrium: a process moves the body in one direction ('downwards'). We say that the cause for this is a physical process we call 'gravitation', and that this process 'acts on the body'.

However, if we immerse the body, ceteris paribus, in different fluid bodies, different mechanical 'behaviors' are observed. In some cases, the body is moved downwards, in others it is moved upwards, and in yet others it is not moved; and, if the body is moved, we observe that its initial acceleration generally differs.

Since the body is the same, and all other conditions being the same, the role of the fluid body in determining the differing behaviors is evident: those behaviors are related to the difference, between the matter of the body and that of the fluid body, in the value of a 'property' we call 'density'. If this difference is positive, the body is moved downwards; if it is zero, the body is not moved; and if it is negative the body is moved upwards.

An interpretation of these observations is the well-known 'Archimedes' Principle' (Archimedes; Halliday et al., 2001): the mechanical behavior of the body is the result of the concurrence, on the body, of two mechanical actions (called 'forces'). One mechanical action is the 'weight of the body', due to 'gravitational interaction' of the body with planet Earth, directed downwards. This action, ceteris paribus, is believed to be proportional to the volume and density of the matter of the body, i.e., proportional to the 'mass of the body'.

The second mechanical action on the body is called 'Archimedes' force' (known also as 'buoyant force' or 'buoyancy'). This action is directed upwards, and it is believed to be directly exerted by the fluid on the body. Its intensity is believed to be proportional to the volume of the fluid body which is 'displaced' by the body, and proportional to the density of the matter constituting the fluid body (i.e., it is believed to be proportional to the 'mass' of the volume of fluid displaced by the body).

According to this interpretation, in the first case (movement downwards), the Archimedes' force is lower in intensity than gravitational action. In the second case (no movement), the intensity of Archimedes' force is equivalent to the intensity of gravitational action, and in the third case (movement upwards) it is higher than the intensity of gravitational action.

Although this interpretation has been 'demonstrated' by the 'Principle' of virtual work (Leroy, 1985), it is very weak from the epistemological point of view because it is based on an assumption. Moreover, it is regarded with serious suspicion because it does not explain the observed decrease in the weight of bodies which sink in fluids and touch the bottom of their containers (Bierman \& Kincanon, 2003; Brown, 1999; Graf, 2004; Jones \& Gordon, 1979; Mohazzabi, 2017; Mungan, 2006; Ray \& Johnson, 1979; Sears, 1963; Taibu, 2015; Taibu et al., 2015). Authors often speak of 'true weight' and 'apparent weight', without reflecting on the fact that terms as 'true' and 'apparent' are the consequences of hypotheses about gravitational action.

Let us examine the current interpretation carefully. Clearly, the existence of the Archimedes' force is conditional 
to the correctness of the hypothesis that the gravitational action on the body is proportional to its mass, unchanged in any kind of fluid in which it is immersed. If we assume that the gravitational action on the body does not change in intensity and direction despite the fluid body in which the body is immersed, to explain the three different mechanical behaviors - downwards, upwards and no movement at all - we are obliged to accept the existence of an opposite concurrent mechanical action on the body, changing in intensity. The existence of this mechanical action is therefore conditional on the correctness of the hypothesis about gravitational mechanical action.

Thus, if we prove that the observed differing mechanical behaviors of the body are due to concurrence, on the body, of the 'weight of the body' and 'Archimedes' force' as we have respectively hypothesized them, then gravitational action on the body is proportional to its mass, unchanged, whatever the nature of the fluid body in which the body is immersed. Instead, if we prove that the behavior of the body is not due to the concurrence of such two mechanical actions, but to a single mechanical action, we necessarily have to call into question the nature of this mechanical action and the hypothesis that what we currently call 'gravitational action' is proportional to mass, unchanged in any kind of fluid body the body is immersed. Therefore, proving that the current interpretation of the phenomen studied by Archimedes is correct or incorrect, is crucial to the debate about gravitation (Cavazzini, 2019).

A line of reasoning has recently been proposed which shows that the current interpretation of the equilibrium of floating is not the only possible interpretation (Cavazzini 2018; 2019). Another interpretation, different from the physical viewpoint, is alternative to the current one, because it is equivalent to the latter from the quantitative viewpoint. According to this alternative interpretation, the observed mechanical behavior of a body completely immersed in a fluid body is not the result of the concurrence of two mechanical actions - the weight of the body and the Archimedes' force (as these two mechanical actions are respectively defined) but the result of only one mechanical action (Cavazzini, 2018; 2019). Therefore, what we currently believe about gravitational mechanical action may be incorrect.

According to this alternative interpretation, a material volume immersed in a fluid body is home to only one mechanical action, the intensity of which, all other conditions being equal, is proportional to the volume and to the difference in density between the matter of the volume and the matter of the fluid which surrounds the volume (Cavazzini, 2018; 2019). The differing mechanical behavior of the material volume when it is immersed in various fluids is the result of changes in the intensity and in the direction of this mechanical action, due to changes in value and sign of the difference in density between the matter of the volume and that of the fluid surrounding it. In substance, the mechanical action in Archimedes' Proposition 6 (Archimedes) is not the result of the concurrence of two mechanical actions.

This mechanical action is not generally proportional to the mass of the immersed material volume, i.e. to the product between the volume and density of the matter constituting that volume. This proportionality occurs only in the particular case when the fluid body in which the material volume is immersed is an ideal vacuum (Cavazzini, 2019).

It has been proposed to interpret this mechanical action as the 'gravitational action on the material volume in that fluid', or the 'weight of the material volume in that fluid', ceteris paribus (Cavazzini 2018; 2019). Ceteris paribus, gravitational mechanical action $W$ on volume $V$ and density $d_{1}$ immersed in the fluid surroundings of density $d_{2}$ is interpreted as:

$$
W=V\left(d_{1} \quad d_{2}\right) w
$$

in which $k$ is a quantity which represents equal conditions.

According to this interpretation, the origin of this mechanical action is the disequilibrium in density between the matter of the body and that of the fluid surrounding that body, i.e., a physical relationship between matter in contact with another matter. Ceteris paribus, a body 'weighs' only if a contrast in density exists between the matter of the body and that of the fluid in which it is immersed. If no disequilibrium in density exists between them, the body is not home to any net gravitational mechanical action. The body is in 'gravitational equilibrium' $(W=0)$.

In the case of a solid volume $V$ floating on the surface of a liquid body, mechanical equilibrium is interpreted as the result of the concurrent action of two opposite 'weights'. If $V-v$ is the part (volume) of the solid immersed in a fluid of density $d_{2}$ (e.g. air) and if $v$ is the part of the solid immersed in a second fluid of density $d_{3}$ (e.g. water), according to equation (1) the mechanical equilibrium of the body is interpreted as: 


$$
(v-v)\left(a_{1}-a_{2}\right)=-v\left(a_{1}-a_{g}\right)
$$

Equation (2) can easily be reduced to:

$$
F\left(d_{2}-d_{\Omega}\right)=v\left(d_{\mathrm{E}}-d_{2}\right)
$$

i.e. the weight in air of the solid volume is equivalent to that in air of a volume of the liquid which is equivalent to that part of the volume of the solid which is immersed in the liquid.

Note that equation (3) represents the current interpretive approach to floating, whereas interpretive approach (2) is markedly different from the physical viewpoint. Since interpretation (3) can be obtained from interpretation (2) and vice-versa (Cavazzini, 2018; 2019), rather paradoxically, these two markedly different cases may be 'indifferently' used from the quantitative viewpoint. This is the reason for the successful use of interpretation (3) over the centuries.

However, the new interpretive approach (equation (2)) is significantly different, because, all other conditions being equal, it hypothesizes that the cause of gravitational mechanical action is a 'physical disequilibrium' between matter which is in contact. As illustrated below, since this is a characteristic of 'spontaneous processes' and as gravitation is also a spontaneous process, this approach relates gravitation to its fundamental nature (Cavazzini, 2019).

\section{Mass as Cause of Gravitation}

Before approaching the main topic of this paper, it is useful to discuss the correctness of the hypothesis that the mass of a body B causes gravitational action on body A in the surroundings of body B.

At present, it seems clear that gravitational action on a body A is related to a second body B in the surroundings of body A. This because, all other conditions being the same, the intensity of the gravitational mechanical action on body A is proportional to the volume and density of the matter of body B (i.e. proportional to the 'mass' of body B). If no body is present in space around a material body, no gravitational action is observed in the latter. Therefore, the existence of another body B seems to be a necessary and sufficient condition to cause gravitation on body A, and the volume and density of the matter of body B concur to determine the intensity of this mechanical action.

Scholars have gone far beyond this concept. They have hypothesized that the mass of second body B is the cause of the gravitational action on a body A (see the definition of 'Gravitation' in the British Dictionary. given above). This is the current belief about gravitational action: gravitational action on a body A is caused by the mass of a second body B in space around body A. The mass of body B 'attracts body A at a distance' ('Newtonian' interpretation; Mach, 1912), or 'warps the four-dimension space-time continuum' in its surroundings (Einstein's interpretation; Einstein, 1916).

However, it is one thing to claim that the intensity of the gravitational action on a material body is proportional to the volume and density of the matter of the second body, and quite another to claim that the product between the volume and density of the matter of body B is the cause of the gravitational action on body A.

To claim that the mass of body B is the cause of gravitation on body A means claiming that body B, due to the simple fact that body B exists in space around body A, causes gravitational mechanical action on body $\mathrm{A}$. Is this true? No, it is not.

To understand this point, let us consider a body A. Let $V_{\mathrm{A}}$ and $d_{\mathrm{A}}$ be the volume and density of the matter of this body, and assume that it is immersed in a fluid 'medium' F. Let $d_{\mathrm{F}}$ be the density of the fluid medium and $d_{\mathrm{A}}>d_{\mathrm{F}}$. Let us now consider a second body B in space around body A, immersed in fluid surroundings $\mathrm{F}$. Let $V_{\mathrm{B}}, d_{\mathrm{B}}$ be the volume and density of the matter of this body. If the mass of body B were the cause of gravitational action on body A, we should always observe a mechanical action on body A, whatever body B is. However, this does not happen, because we observe a mechanical action on body A only if the difference $d_{\mathrm{B}}-d_{\mathrm{F}}$ is not 0 .

Let us note that, although body B 'has mass', no net mechanical action on body $\mathrm{A}$ is observed if the difference $d_{\mathrm{B}}$ - $d_{\mathrm{F}}$ is 0 . This can be interpreted as currently, but it may also indicate that the origin of the mechanical action of body B on body A is a process related to the disequilibrium in density between the matter constituting body B and that which constitutes the fluid in which the two bodies are immersed.

The actual cause of gravitational action on body A could be not the mass of body B, but the disequilibrium in 
density between the matter of body B and that of the fluid body in which the two bodies are immersed. In other words, the mechanical action caused by body $\mathrm{B}$ on body A could be not proportional to product $V_{\mathrm{B}} \cdot d_{\mathrm{B}}$ between the volume and density of the matter of body $\mathrm{B}$, but proportional to product $V_{\mathrm{B}}\left(d_{\mathrm{B}}-d_{\mathrm{F}}\right)$ (Cavazzini, 2019).

If, however, $d_{\mathrm{F}}$ is close to 0 or if it is negligible with respect to $d_{\mathrm{B}}$, the quantity $V_{\mathrm{B}}\left(d_{\mathrm{B}}-d_{\mathrm{F}}\right)$ is close to quantity $V_{\mathrm{B}} \cdot d_{\mathrm{B}}$. This could be the cause of the current misconception: gravitational action on a body in relation to a second body is not generally proportional to the mass of the second body. However, this does occur if the fluid in which the two bodies are immersed is characterized by a density value of 0: i.e. the 'ideal vacuum' (Cavazzini, 2019).

Thus, the cause of the gravitational action on one body could be not the mass of a second body but the disequilibrium in density between the matter of the second body and that of the fluid body in which the bodies are immersed.

However, this is a necessary but not sufficient condition. Even if disequilibrium in density exists between the matter of body B and that of the fluid, body A is not home to any gravitational action if there is no disequilibrium in density between the matter of body $\mathrm{A}$ itself and that of the fluid, i.e., if difference $d_{\mathrm{A}}-d_{\mathrm{F}}$ is 0 . Gravitational action on body A occurs only if the matter of body B and that of body A are both in disequilibrium from the viewpoint of their density with the matter of the fluid surroundings. Given two bodies, either both are home to gravitational mechanical action, or neither of them are.

If the cause of the gravitational process between bodies is the disequilibrium in density between matter which is in contact, the direction of the mechanical action is not necessarily that of an 'attraction' between the bodies: it may also be that of a 'repulsion', depending on the sign of the differences in density among body A, body B, and the fluid body, respectively. For example, if $d_{\mathrm{A}}-d_{\mathrm{F}}>0$ and $d_{\mathrm{B}}-d_{\mathrm{F}}<0$, the gravitational action on the two bodies is in the direction of a reciprocal 'repulsion' (Cavazzini, 2019).

Nevertheless, in a gravitational process, the bodies neither attract each other nor repulse each other. We usually say that gravitation is 'attractive' because we are looking at the movement of the bodies in their initial positions. However, this is a misconception. Gravitation is a spontaneous process, and the cause and the aim of gravitation are similar to those of any other spontaneous process in the Universe, as we shall now illustrate.

\section{Spontaneous Processes}

In the world surrounding us, we very frequently observe phenomena which occur 'spontaneously'. This word is generally used precisely to indicate such kinds of processes, meaning that they start and take place 'alone'; by the word 'process' we mean that we observe something changing.

We can easily give examples of these phenomena, in both physical and chemical sciences. In Physics, the spontaneous process we interpret as 'heat transfer between bodies at different temperature' is well known. When bodies characterized by different temperatures are in contact, we observe changes in the values of some properties of the matter constituting these bodies.

Another very well-known example of spontaneous process is that which occurs when masses of gas at different pressures are in contact: changes in the values of the properties of the gaseous systems are observed.

The process of 'consuming' energy of motion is a very frequent spontaneous process in daily life. If bodies which are in contact move at different velocities in the same reference frame, we observe a spontaneous process called 'friction'. Another well-known process, in Physics, is the spontaneous propagation of light from a source within a medium.

In Chemistry, the solution of common salt in water is a well-known example of a process which occurs spontaneously when water and crystals of salt are in contact.

The above examples show interesting analogies. Let us consider the first three: matter (bodies) in contact is characterized by a disequilibrium of some kind.

The spontaneous process we call 'heat transfer' occurs when matter in contact is in disequilibrium from the viewpoint of the 'property' of the matter called 'temperature'. The second spontaneous process occurs when gaseous matter in contact is in disequilibrium from the viewpoint of the property called 'pressure'. The third process, 'friction', takes place when matter in contact is in disequilibrium with the property we call 'velocity in a frame'.

In all these examples, matter is (i) in contact and (ii) in disequilibrium from the viewpoint of one of their 'properties'. In the first example, the property is 'temperature', in the second, it is 'pressure', in the third, the property is 'velocity in a frame'. 
We emphasize that all these properties of matter are what we call 'intensive': they are related to 'qualities' of matter, and the value of each given property may be considered as a quantitative 'index' of a certain 'quality'.

The property of the matter in relation to the 'quantity' of that matter is called 'extensive' - we call it 'volume'. Its value is proportional to its quantity. However, this is only true if all the qualities of the matter are fixed. 'Temperature' is a property of matter, 'volume' is a property of 'bodies', and bodies' are quantities of matter.

We observe that processes occur spontaneously only when matter in contact is in disequilibrium from the viewpoint of one or more of its 'qualities'. If matter in contact is in equilibrium from this viewpoint, we do not observe any change in the material systems involved. We do not observe any 'process'.

\section{Characteristics of Spontaneous Processes}

The 'process', i.e., the changes we observe, are changes in the values of 'measurable properties' of the matter in question. In particular, we observe changes in the values of the intensive property the disequilibrium of which appears to be at the origin of the process itself. For example, in the spontaneous process we interpret as 'heat transfer', the value of the temperature of the matter characterized by the lower value increases with time, whereas that of the temperature of the matter characterized by the higher value of temperature decreases with time.

The same kind of behavior occurs in the case of the spontaneous process which takes place when two masses of gas in contact are characterized by differing pressure values. Pressure increases in the gaseous system characterized by the lower pressure value, and pressure decreases in the gaseous system characterized by the higher value. This behavior is also typical of friction: velocity increases in matter with the lower velocity and decreases in matter with the higher velocity.

As the contrast in the value of the property decreases, progressive slowing down in the changes can be observed in the material systems involved. When the contrast is close to zero, these changes are no longer observable, i.e., the process 'stops'. This is why we say that the disequilibrium in the value of the property is at the origin of the process.

Below, we list the characteristics of the spontaneous processes:

1) A spontaneous process originates from matter which is in contact and in disequilibrium from the viewpoint of one (or more) of its 'qualities'.

2) Each quality of the matter is related with one 'intensive' property of that matter.

3) The value of the intensive property is the quantitative index of the quality. Disequilibrium in the value of the intensive property means disequilibrium in the quality.

4) A spontaneous process reduces or tends to reduce the disequilibrium in the value of the property. We can therefore say that a spontaneous process occurs in the direction of the reduction of the disequilibrium in the quality between the matter involved.

5) The process stops as soon as equilibrium in the value of the property is reached between the matter involved.

By 'stops' we mean that changes can no longer be observable, although this does not mean that reciprocal physical action between the matter ceases. Indeed, the physical action which matter in contact exerts on another continues, but there is no net physical action of one matter on the other.

\section{Gravitation as a Spontaneous Process}

Gravitation is a spontaneous physical process. Therefore, if analysis on the spontaneous processes proposed above is correct, gravitation should show the characteristics observed for other spontaneous processes:

a) The origin of the gravitational process should contain matter in contact which is in disequilibrium from the viewpoint of one 'quality'.

b) This quality should be related to an intensive property of the matter.

c) The gravitational process - i.e., the 'weight' of the body, and its movement - should occur in the direction which reduces the difference in the value of the property between the matter in contact.

d) The gravitational process should stop - i.e., no movement of the body should occur, and no weight of the body may be measured - when equilibrium is reached in the value of the property between the matter in contact.

Since

1) Gravitational action on a body is observed (i.e., the body is moved and a 'weight' of the body is measured) if 
disequilibrium in density exists between the matter of the body and that of the fluid body in contact with the body, and no gravitational action on a body is observed (i.e., the body is not moved, and no 'weight' of the body is measured) if there is no disequilibrium in density between the matter of the body and that of the fluid surrounding it;

2) The matter of the body and that of the fluid surroundings are in mutual contact;

3) Density is an intensive property of the matter;

4) The process - i.e.. the movement and 'weight' of the body - occurs in the direction which reduces the disequilibrium in the value of the property between the matter of the body and that of the fluid surroundings of the body (downwards or upwards);

5) The gravitational process stops - i.e., the body is no longer moved, and no 'weight' of the body is measured when equilibrium in the value of the property density is reached between the matter of the body and that of the fluid surroundings of the body.

we can infer that the gravitational process shows all the characteristics which are typical of other spontaneous processes.

Therefore, as for other spontaneous processes, we hypothesize that the origin of the gravitational process on a material body is the disequilibrium, between the matter of the body and that of the fluid surrounding the body, in a quality of the matter which is related to the intensive property which we call 'density'.

\section{Cause of a Spontaneous Process}

In Section 4. and 5., we argued that a spontaneous process occurs when matter in contact is in disequilibrium from the viewpoint of one of its 'qualities'. Since no process takes place if there is no disequilibrium between the matter, and since the matter is in contact, we hypothesize that a spontaneous process originates from the disequilibrium, between the matter, in its capacity to undertake a certain particular kind of 'action' on contact (i.e., on its surroundings).

If matter $\mathrm{A}$, which, in a certain time-span, is capable of undertaking a certain kind of action at its contact, is surrounded by matter B which has a different capability to do the same kind of action (matter B is the surroundings of matter A and vice-versa), a net action takes place between matter A and matter B. This results in changes in the matter involved ('process').

In other words, we interpret a spontaneous process as the result of the differing mutual capability of matter to act in a certain way on each other. According to this interpretation, the process 'stops' when equilibrium is reached, between the matter in question, in its capability to undertake the action related with quality. When there is no net action between them, no change occurs in the matter (the process 'stops').

According to this interpretation, whenever we observe a spontaneous process, we should realize that what we observe is related to disequilibrium, between matter which is in contact, in (at least) one of its 'qualities'. Each quality of the matter is related to an intensive property of that matter, and to a capability of the matter to undertake an action of a certain kind on its surroundings. In the case of the gravitational process, the intensive property is 'density'.

It should also be recalled that, when equilibrium in quality is reached between the matter in contact, and the process 'stops', matter continues the action which is in relation with quality, if that action is due to a 'proper' quality of that matter. In other words, by its very existence, matter performs a continuous action on its surroundings. Instead, a spontaneous process is observed only if a net action occurs between the matter and its surroundings.

The kind of action may be 'clear', as in the case of the three examples of spontaneous processes described above to illustrate the fundamental characteristics of these processes, because they are evidently related to microand/or macroscopic mechanical disequilibria between matter which is in contact.

Instead, in the case of gravitation, this interpretation is not so immediately apparent, because the cause of the gravitational process on body A is presently believed to be related to the 'action' of body B in the surroundings, the mass of which 'attracts' body A at a distance, or warps the four-dimension space-time continuum at a distance. In substance, it is currently believed that the action of a distant mass is the cause of the gravitational process.

Although it is clear that body B in the surroundings of body A plays a role in originating a gravitational action on body A, what kind of role it plays is not so clear. All other conditions being the same, extensive experimental 
evidence indicates that the intensity of the gravitational mechanical action on body A depends on the volume and density of the matter constituting body B (i.e.,it depends on the mass of body B).

However, in Section 3. we showed that the origin of gravitation on body A could be not the mass of body B, but the (simultaneous) disequilibrium in density between body A and the fluid surroundings and, respectively, between body $\mathrm{B}$ and the fluid surroundings.

Quantity $V_{\mathrm{B}} \cdot d_{\mathrm{B}}$ could not play a role in determining the intensity of the gravitational mechanical action on body A: it could be quantity $V_{\mathrm{B}}\left(d_{\mathrm{B}}-d_{\mathrm{F}}\right)$. And, vice-versa, it could be not quantity $V_{\mathrm{A}} \cdot d_{\mathrm{A}}$ which plays a role in determining the intensity of gravitational action on body B: it could be quantity $V_{\mathrm{A}}\left(d_{\mathrm{A}}-d_{\mathrm{F}}\right)$.

Modeling the value of the intensity of gravitational mechanical action in terms of quantities $V_{\mathrm{A}}\left(d_{\mathrm{A}}-d_{\mathrm{F}}\right)$ and $V_{\mathrm{B}}\left(d_{\mathrm{B}}-d_{\mathrm{F}}\right)$ will be the subject of a forthcoming paper. We anticipate here only an important characteristic of spontaneous processes: during a spontaneous process, the disequilibrium in the capability of the matter to undertake the particular action (i.e.the disequilibrium in the value of the property related to the particular action) is progressively reduced, and a different kind of disequilibrium appears. In the meantime, disequilibrium of a certain kind is reduced/eliminated, and disequilibrium of a different kind is generated.

For example, in the spontaneous process we call 'heat transfer between matter at a different temperature', the disequilibrium in temperature - we usually say the 'thermal' disequilibrium - is progressively reduced/eliminated. In the meantime, disequilibrium of a different kind (mechanical) is generated, because, as the temperature in the matter changes, so there are changes in the volumes of matter, and these changes correspond to mechanical disequilibrium. The emergence of disequilibrium of a different kind (in this case, 'mechanical') during the 'parent' disequilibrium (in this case, 'thermal') is reduced/eliminated, appears to be a characteristics of spontaneous processes, and is functional for reducing/eliminating the parent disequilibrium.

Likewise, in the case of the gravitational process, there is the emergence of disequilibrium of a different kind (mechanical i.e., the 'weight' of the body) and this kind of disequilibrium is functional to reducing/eliminating the disequilibrium in density (i.e., the parent disequilibrium) between the matters in contact.

\section{Conclusions}

All spontaneous processes originate from matter in contact which is in physical disequilibrium. Gravitation is a spontaneous physical process, and the cause of this physical process is a particular physical disequilibrium between matter which is in contact.

Viewing gravitation as a spontaneous process shows both the causal reason and the final reason of this process, which we call its 'aim'. Just as the process we call 'heat transfer' is the spontaneous process by which the Universe reduces/eliminates disequilibria existing between its parts due to differences in the 'quality' of the matter related to the property of the matter we call 'temperature', the process we call 'gravitation' is the spontaneous process by means of which the Universe reduces/eliminates disequilibria between its parts, due to differences in the quality of the matter related to the property of the matter we call 'density'. This is achieved by moving bodies.

Ceteris paribus, a body is moved in the direction which depends on the sign of the difference in density between the matter of the body and that of its fluid surroundings.

If the movement of the bodies concerns their initial positions, gravitational action seems to be reciprocally attractive or repulsive. However, this is illusory. Gravitation is neither attractive, nor repulsive, because bodies cannot de facto attract or repulse each other. The 'aim' of the action is to reduce/eliminate the disequilibrium in density between the matter in question, and the direction in which the bodies are moved aims at achieving this aim.

At present, Einstein's General Theory of Relativity is considered the best interpretation of gravitation. According to this theory, mass is the cause of this physical process, which exerts gravitation at a distance on other bodies by deforming a four-dimension mathematical continuum in its surroundings, which has been called the 'space-time continuum' (Einstein, 1916). In substance, according to Einstein's hypothesis, matter (mass) determines disequilibria in space-time, and gravitation takes origin from these disequilibria.

However, gtavitation is a spontaneous process, and in this study we have shown that a spontaneous process originates from the disequilibrium of matter which is in contact from the viewpoint of one of its qualities/properties, and space and time are not physical properties of the matter. We have also shown (Section 3.) that the mass of one body could be not the cause of this physical process on another body: the actual cause could be disequilibrium, between matter in contact, in the quality of the matter which is related to the intensive 
property of the matter we call 'density'. Therefore, with regard to the cause of the gravitational process, this interpretation significantly differs from that given by General Theory of Relativity.

Viewing gravitation as a spontaneous process significantly moves our viewpoint about this physical process. A different physical interpretation of the 'Archimedes' Principle' shows that the mechanical action on one body A, which we call 'gravitation', could be not generally proportional to the quantity we call the 'mass' of body A, nor it could be proportional to the mass of body B which we believe exerts gravitational action on body A.

All other conditions being the same, gravitational action on a body could be proportional to the volume of that body and to the disequilibrium in density between the matter of the body and that of the fluid body in which the body is immersed. The main cause of this physical process could be this physical disequilibrium, and the aim of the process is to reduce/eliminate this disequilibrium.

\section{Acknowledgments}

This work was financed by C.N.R. Istituto di Geoscienze e Georisorse. The author is very grateful to mother-tongue technical translator Ms. Gabriel Walton for her generous care in language revision.

\section{Conflict of interests}

The authors declare that there is no conflict of interests regarding the publication of this paper.

\section{References}

Archimedes of Syracuse. On floating bodies, Book I (in Italian, in Opere di Archimede; Torino, UTET, 1974).

Bierman, J., \& Kincanon, E. (2003). Reconsidering Archimedes' Principle. The Physics Teacher, 41, 340-344. https://doi.org/10.1119/1.1607804

Brown, R. (1999). Weight - don't use the word at all. The Physics Teacher, 37, 241. https://doi.org/10.1119/1.880241

Cavazzini, G. (2018). A New Physical Interpretation of Archimedes' Principle. Journal of Applied Mathematics and Physics, 6, 215-223. https://doi.org/10.4236/jamp.2018.61020

Cavazzini, G. (2019). Archimedes' Principle and the Concept of Gravitation. Applied Physics Research, 11/6, 9-18. https://doi.org/10.5539/apr.v11n6p9

Einstein, A. (1916). Uber die spezielle und allgemeine Relativitatstheorie (gemeinverstndlich), Vieweg, Braunschweig (in Italian, Relatività. Esposizione divulgativa, Torino, Universale Bollati-Boringhier, 1988).

Graf, E. H. (2004). Just what did Archimedes say about buoyancy? The Physics Teacher, 42, 296-299. https://doi.org/10.1119/1.1737965

Halliday, D., Resnick, R., \& Walker, J. (2001). Fundamentals of Physics (6th ed.). New York, Wiley.

Jones, G. E., \& Gordon, W. P. (1979). Removing the buoyant force. The Physics Teacher, 17, 59-60. https://doi.org/10.1119/1.2340127

Leroy, B. (1985). Archimedes' Principle: A simple derivation. European Journal of Physics, 6, 56.

Mach, E. (1912). Die Mechanikin ihrer Entwickelung Historisch-Kritish Dargestellt (7th ed.) (in Italian, La meccanica nel suo sviluppo storico-critico, Torino, Bollati-Boringhieri, 1977).

Milgrom, M. (1983a). A modification of the Newtonian dynamics as a possible alternative to the hidden mass hypothesis. Astrophysical Journal, 270, 365-370. https://doi.org/10.1086/161130

Milgrom, M. (1983b). A modification of the Newtonian dynamics - Implications for galaxies. Astrophysical Journal, 270, 371-389. https://doi.org/10.1086/161132

Moffat, J. W., \& Rahvar, S. (2013). The MOG weak field approximation and observational test of galaxy rotation curves. Monthly Notices of the Royal Astronomical Society, 436(2), 1439-14511-89. https://doi.org/10.1093/mnras/stt1670

Mohazzabi, P. (2017). Archimedes' Principle revisited. Journal of Applied Mathematics and Physics, 5, 836-843. https://doi.org/10.4236/jamp.2017.54073

Mungan, C. E. (2006). What is the buoyant force on a block at the bottom of a beaker of water? Forum on Education of The American Physical Society, Spring 2006 Newsletter. https://www.aps.org/units/fed/newsletters/spring2006/mungan.html 
Newton, I. (1687). Philosophiae Naturalis Principia Mathematica (1st ed.). London, Societatis Regiae ac Typis, Joseph Streater.

Newton, I. (1693). Letters to doc. R. Bentley. Four letters from Sir Isaac Newton to R. Bentley, containing some arguments in proof of a deity (1693). London, R. and J. Dodslet (1752).

Ray, J. R., \& Johnson, E. (1979). Removing the buoyant force, a follow-up. The Physics Teacher, 17, 392-393. https://doi.org/10.1119/1.2340279

Sears, F. W. (1963). Weight and weightlessness. The Physics Teacher, 1, 20-23. https://doi.org/10.1119/1.2350557

Taibu, R. (2015). Terms vs, concepts - the case of weight. The Physics Teacher, 55, 34-35. https://doi.org/10.1119/1.4972495

Taibu, R., Rudge, D., \& Schuster, D. (2015). Textbooks presentations of weight: Conceptual difficulties and language ambiguities. Physical Review Education Research, 11, 1-20 https://doi.org/10.1103/PhysRevSTPER.11.010117

\section{Copyrights}

Copyright for this article is retained by the author(s), with first publication rights granted to the journal.

This is an open-access article distributed under the terms and conditions of the Creative Commons Attribution license (http://creativecommons.org/licenses/by/4.0/). 